\title{
AC loss measurements in HTS coil assemblies with hybrid coil structures
}

\author{
Zhenan Jiang ${ }^{1}$, Nicholas J. Long ${ }^{1}$, Mike Staines ${ }^{1}$, Rodney A. Badcock ${ }^{1}$, Chris W. \\ Bumby $^{1}$, Robert G. Buckley ${ }^{1}$, Naoyuki Amemiya ${ }^{2}$ \\ ${ }^{1}$ Robinson Research Institute, Victoria University of Wellington, PO BOX 33436, Petone, \\ Lower Hutt 5046, New Zealand \\ ${ }^{2}$ Department of Electrical Engineering, Graduate School of Engineering, Kyoto University, \\ Kyoto-Daigaku-Katsura, Nishikyo, Kyoto 615-8510, Japan \\ E-mail:zhenan.jiang@vuw.ac.nz
}

\begin{abstract}
Both AC loss and wire cost in coil windings are critical factors for HTS AC machinery applications. We present AC loss measurement results in three HTS coil assemblies at $77 \mathrm{~K}$ and $65 \mathrm{~K}$ which have hybrid coil structure comprising one central winding $(\mathrm{CW})$ and two end windings (EWs) wound with ReBCO and BSCCO wires with different self-field $I_{\mathrm{c}}$ values at $77 \mathrm{~K}$. All AC loss results in the coil assemblies are hysteretic and the normalized AC losses in the coil assemblies at different temperatures can be scaled with the $I_{\mathrm{c}}$ value of the coil assemblies. The normalised results show that AC loss in a coil assembly with BSCCO CW can be reduced by using EWs wound with high $I_{\mathrm{c}}$ $\mathrm{ReBCO}$ wires, whilst further AC loss reduction can be achieved by replacing the BSCCO CW with ReBCO CW. The results imply a flexible hybrid coil structure is possible which considers both AC loss and wire cost in coil assemblies.
\end{abstract}

Keywords: HTS coil assemblies, AC loss, Double pancake coil

\section{Introduction}

AC loss is a major issue for AC devices using HTS conductors e.g. transformers, motors/generators, and DC magnets with field ramping. Both BSCCO and ReBCO commercial HTS wires are good candidates for these applications [1-6]. Extensive experimental and numerical investigations have been undertaken to characterise the AC loss in coils and coil assemblies wound with BSCCO or ReBCO conductors but never with mixed conductors nor conductors with different critical current performance [2-5, 7-20].

As the schematic view in figure 1 of a HTS coil assembly shows, conductors in different parts of a assembly experience different magnetic fields: conductors in the end turns are exposed to larger perpendicular magnetic field components; conductors in the central windings are primarily exposed to parallel magnetic field components. Experimental and numerical studies demonstrate that the AC loss in coils wound with BSCCO wire increases with the $2^{\text {nd }}$ to $3^{\text {rd }}$ power of $I_{t} / I_{c}$, coil [2] while AC loss in coils wound with ReBCO wire increases with the $3^{\text {rd }}$ to $4^{\text {th }}$ power of $I_{\mathrm{t}} / I_{\mathrm{c} \text {, coil }}$ where $I_{\mathrm{t}}$ is the amplitude of the AC transport current, and $I_{\mathrm{c} \text {, coil }}$ is the coil critical current [10-16]. These observations suggest that an approach employing hybrid winding assemblies may reduce AC loss and/or total wire cost: 1) using high $I_{\mathrm{c}} \mathrm{ReBCO}$ wire in the end-turns to reduce AC loss by increasing $I_{\mathrm{c} \text {, coil }}$; 2) cheaper low $I_{\mathrm{c}}$ ReBCO wire in the central part of the assemblies as the magnetic field is largely parallel hence lowering AC loss, or 3) using BSCCO wires in the central part of the assemblies to reduce total wire cost. A related approach was used in a HTS DC magnet where high $I_{\mathrm{c}}$ wire was used to increase $I_{\mathrm{c} \text {, coil }}$ and hence increase the magnetic field generated [21]. However, there has been no report of AC loss measurements on hybrid windings e.g. using two wire technologies. 
In this paper, we report AC loss measurements on three HTS coil assemblies comprising a stack of six double pancake coils wound with BSCCO and ReBCO wires with different self-field $I_{\mathrm{c}}$ values at $77 \mathrm{~K}$. The coil assembly was split into a central winding and two identical end windings as illustrated in figure 2 . The central winding (CW) comprised four double pancake coils (DPC) and each end winding (EW) comprised one DPC. Multiple central and end coils were fabricated using both BSCCO and ReBCO wires. The $I_{\mathrm{c}}$ and AC loss at $77 \mathrm{~K}$ and $65 \mathrm{~K}$ were measured for coil assemblies with the same wire technology and with mixed BSCCO and ReBCO windings: Assemblies of, all BSCCO or ReBCO coils, and of mixed wire types were studied i.e. in the latter cases EW and CW employing alternately BSCCO and ReBCO coils were studied. The AC loss of each assembly is compared. 


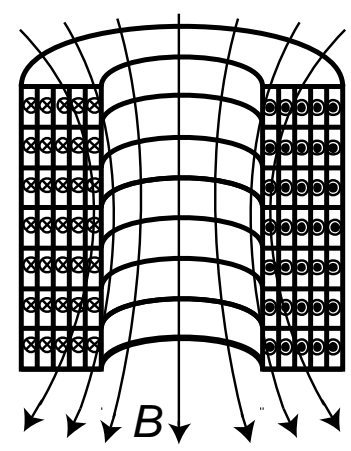

Figure 1. Schematics of magnetic field distribution in an HTS magnet

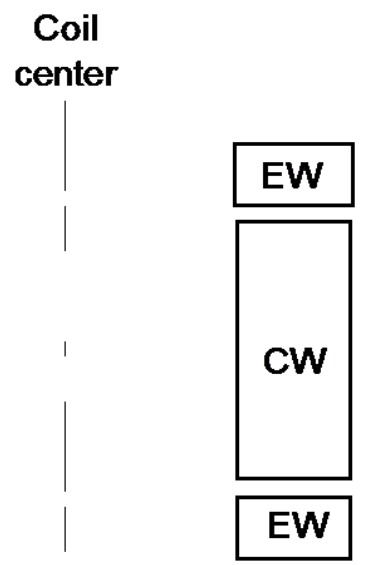

Figure 2. The schematic of HTS assembly

\section{Experimental methods}

\subsection{HTS coil assemblies}

All coils were manufactured as double pancakes by HTS-110 Ltd [5]. The coils are dry-wound and are not resin encapsulated although turn-to-turn insulation is supplied by co-wound $0.05 \mathrm{~mm}$ thick Nomex paper. Table 1 lists the specifications of the wires used in the coil windings.

The first coil assembly comprises a BSCCO central winding (CW) and two BSCCO end windings (EW) referred to as the All-BSCCO assembly. Both CW and EWs were wound with AMSC wire with an average self-field $I_{\mathrm{c}}$ of $156 \mathrm{~A}$ at $77 \mathrm{~K}$ and wire dimensions $4.3 \mathrm{~mm}$ wide and $0.3 \mathrm{~mm}$ thick. The $\mathrm{CW}$ was made up of a stack of four double pancake coils (DPCs). Each DPC had 40 turns, a height of $9 \mathrm{~mm}$, and coil ID (inner diameter) and $\mathrm{OD}$ (outer diameter) are 60 and $73 \mathrm{~mm}$ respectively. The height of the $\mathrm{CW}$ was $38 \mathrm{~mm}$ including $1.5 \mathrm{~mm}$ thick G-10 rings at each end. Neighbouring DPCs were connected using ten BSCCO strips (see figure 4a) in parallel to reduce contact resistance arising from soldering joints. Sumitomo DI-BSCCO Type H wire with an self-field $I_{\mathrm{c}}$ of $170 \mathrm{~A}$ was used for the BSCCO strips and soldering between the BSCCO strips and the ends of the DPCs was done using low melting point 66.7In33.3Bi solder at $200{ }^{\circ} \mathrm{C}$. The two EWs comprised of one DPC. The gap between the CW and EW is $1.5 \mathrm{~mm}$. Figure 3 shows the $\mathrm{CW}$ and EWs before assembly into a coil stack.

The second coil assembly has a BSCCO/ReBCO hybrid structure. It comprises a BSCCO CW and two ReBCO EWs referred to as the BSCCO/ReBCO assembly. The BSCCO CW used was the same as that used in the All-BSCCO assembly. ReBCO wire for the EWs is $4.0 \mathrm{~mm}$-wide and $0.1 \mathrm{~mm}$-thick SuNam wire (HCN04200) with an average self-field $I_{\mathrm{c}}$ of $238 \mathrm{~A}$ at $77 \mathrm{~K}$ [22]. Note the $I_{\mathrm{c}}$ of the ReBCO wire for the EW is much larger than that of BSCCO wire. The wire was selected to give reduced $I_{t} / I_{\mathrm{c} \text {, coil }}$ and in turn to reduce AC loss in the EW where the largest perpendicular magnetic field component is observed. Each ReBCO DPC has a nominal external dimension that was identical to the BSCCO DPCs; additional layers of Nomex paper are incorporated radially between turns and axially between the two layers of the ReBCO DPCs to compensate for 
the reduced thickness and width of the ReBCO wire relative to the BSCCO wire.

The third coil assembly has a ReBCO/ReBCO hybrid structure. It comprises $\mathrm{ReBCO} C W$ wound with SuperPower wire (SCS 4050) [23] and two ReBCO EWs disassembled from the second coil assembly. We refer to this assembly as the All-ReBCO assembly. The ReBCO wire for the $\mathrm{CW}$ is $4.0 \mathrm{~mm}$-wide and $0.1 \mathrm{~mm}$ thick wire with a self-field $I_{\mathrm{c}}$ of $86 \mathrm{~A}$. Note the $I_{\mathrm{c}}$ of the ReBCO wire for the $\mathrm{CW}$ is much less than that of the $\mathrm{ReBCO}$ wire for the EW. This option was chosen because the magnetic field experienced by the conductors in the CW is essentially parallel to the wide face of the HTS tape unlike the conductors in the EW, hence cost can be reduced by using wire with lower $I_{\mathrm{c}}$.

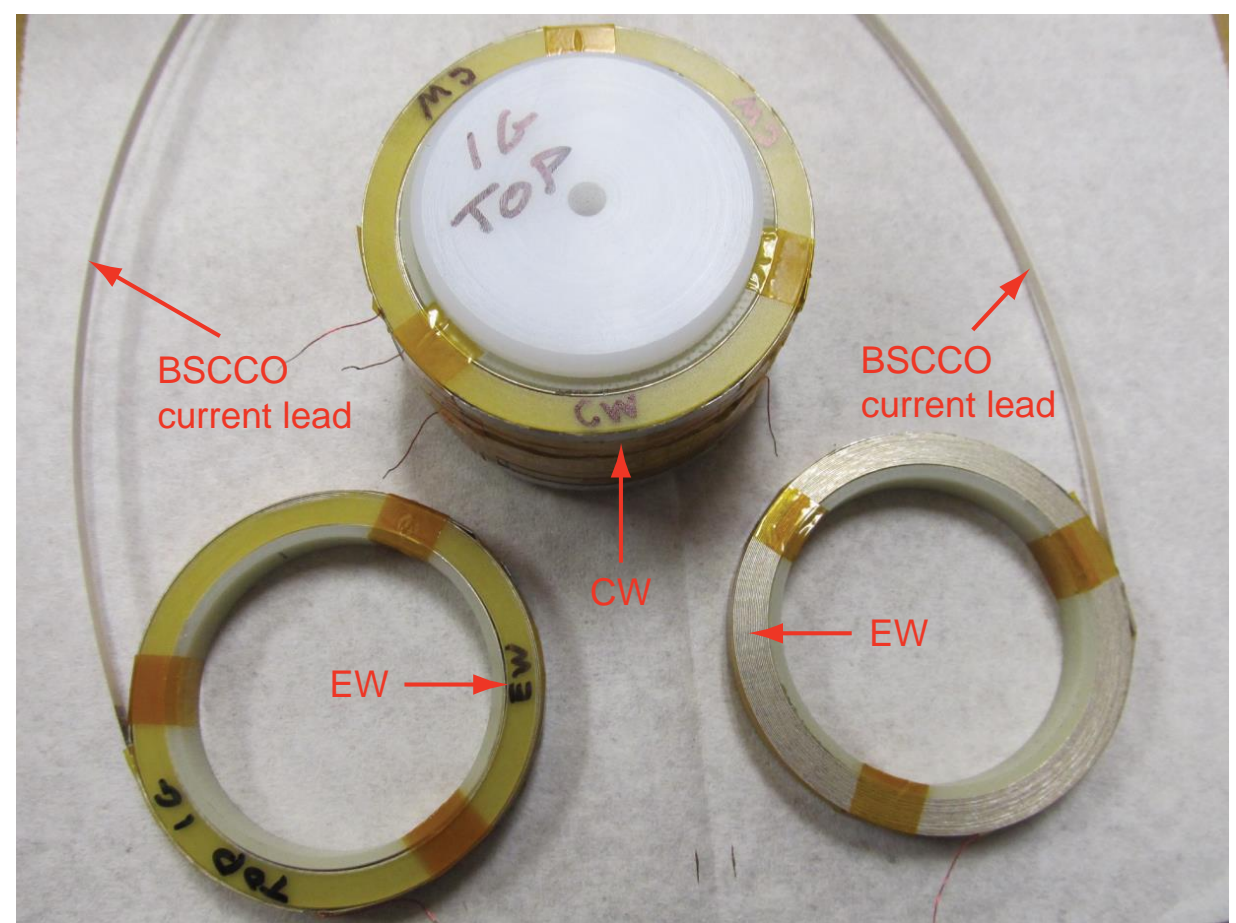

Figure 3. Photos of the components of the All-BSCCO assembly: the CW comprising four stacks and two EWs wound with $\mathrm{BSCCO}$ wire

Table 1 BSCCO and ReBCO wire specifications

\begin{tabular}{cccc}
\hline Wire type & BSCCO & ReBCO for EW & ReBCO for CW \\
Manufacturer & AMSC & SuNAM & SuperPower \\
Critical current (A) & 156 & 238 & 86 \\
Width (mm) & 4.3 & 4.0 & 4.0 \\
Thickness (mm) & 0.3 & 0.1 & 0.1 \\
Substrate & N.A. & Non magnetic & Non magnetic \\
\hline
\end{tabular}

2.2 Measurement method

Figure 4(a) shows the assembled All-BSCCO assembly. Measurements were carried out at $77 \mathrm{~K}$ and 65 
K. PT100 thermometers were mounted on the upper and lower flanges of the coils to monitor the liquid nitrogen $\left(\mathrm{LN}_{2}\right)$ temperature. BSCCO wires were used as current leads for the coil assemblies. Voltage taps were attached in the end of the coils for both $I_{\mathrm{c} \text {, coil }}$ and AC loss measurements. The coil assemblies were put into a double-wall structured $\mathrm{LN}_{2}$ dewar as shown in figure 4(b) [24]. The temperature of the $\mathrm{LN}_{2}$ was varied between $77 \mathrm{~K}$ and $65 \mathrm{~K}$ by reducing the pressure in the dewar.

The AC loss measurement equipment is schematically shown in figure 5 that uses two lock-in amplifiers (Stanford 830): one for measuring the current amplitude and phase; the other for registering the in-phase voltage from the coil voltage taps. The lock-in amplifier internal oscillator was used as the input signal for a CROWN K2 audio amplifier. The coils were energised through a toroidal transformer (TT) immersed in a $\mathrm{LN}_{2}$ dewar. A capacitor bank was connected to the primary of the toroidal transformer for impedance matching. A two-stage current transformer (CT) was used as a phase reference of the coil current.

All three coil assemblies had a measured inductance of approximately $2.4 \mathrm{mH}$ at $77 \mathrm{~K}$. An attenuator with the attenuation ratio of 51:1 was used for the coil voltage output because of the input voltage limit of $1 V_{\text {rms }}$ in the lock-in amplifier. There is a possibility of phase shifts resulting from the introduction of the attenuator. To investigate the phase shift effect of the attenuator, transport AC loss in a long straight ReBCO wire (SCS 4050) with an $82 \mathrm{~cm}$ - distance between the voltage taps was measured with/without the attenuator. The relatively long wire-length was chosen to improve $\mathrm{S} / \mathrm{N}$ ratio. The ReBCO wire has a self-field $I_{\mathrm{c}}$ of $110 \mathrm{~A}$ at $77 \mathrm{~K}$.

Figure 6 displays the measured transport $\mathrm{AC}$ loss at $77 \mathrm{~K}$ plotted as a function of the transport current normalised by wire $I_{\mathrm{c}}$. As can be seen in the figure the measured loss using the attenuator agrees well with the loss measured when not using the attenuator. The result indicates the attenuator does not introduce a significant phase error. Therefore coil assembly AC loss was measured using the attenuator without a compensating phase shift. A calculation also confirmed that the phase shift due to the attenuator's resistive loading of the HTS assembly inductance as well as those due to cable capacitance are less than $0.01^{\circ}$ which is the resolution of the lock-in amplifiers used.

The AC loss per cycle of a HTS coil assembly can be given as,

$$
Q=\frac{V_{\mathrm{rms}} I_{\mathrm{coil}, \mathrm{rms}}}{f} .
$$

where, the $V_{\text {rms }}$ is the rms value of in-phase voltage from voltage taps attached in the ends of the coil assembly with regard to the phase of the coil current, $I_{\text {coil, rms }}$ is the rms value of the coil current, and $f$ is the frequency of the coil current. 


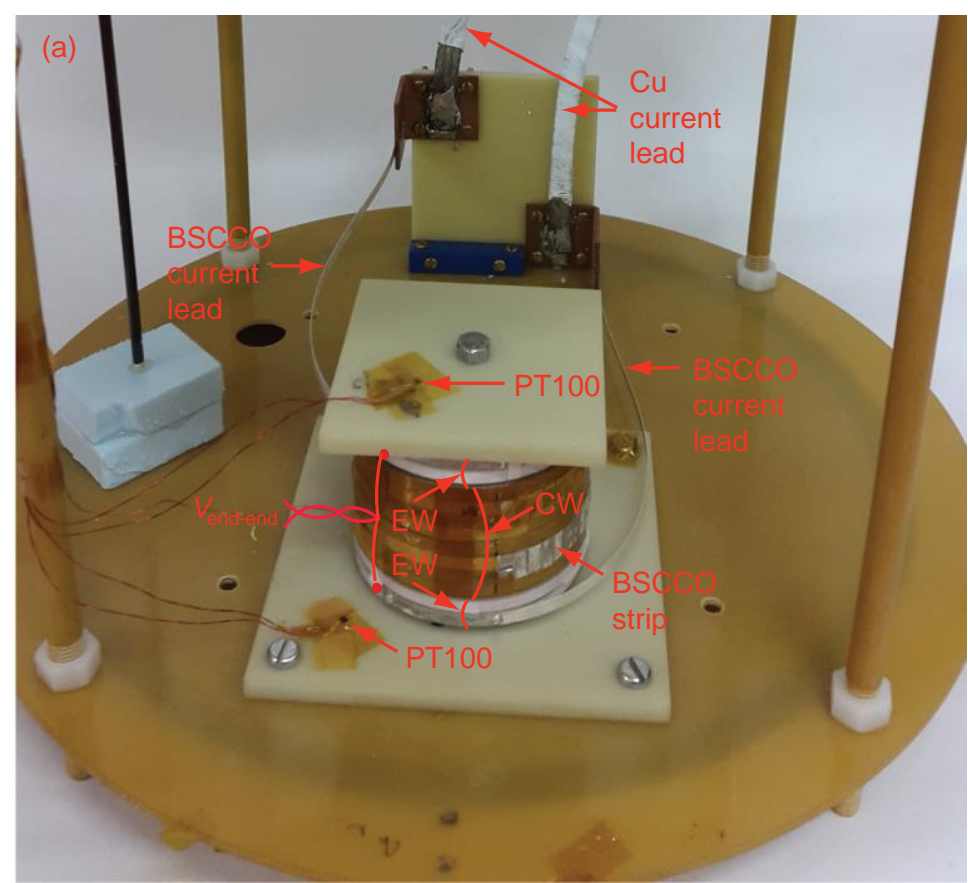

(b)

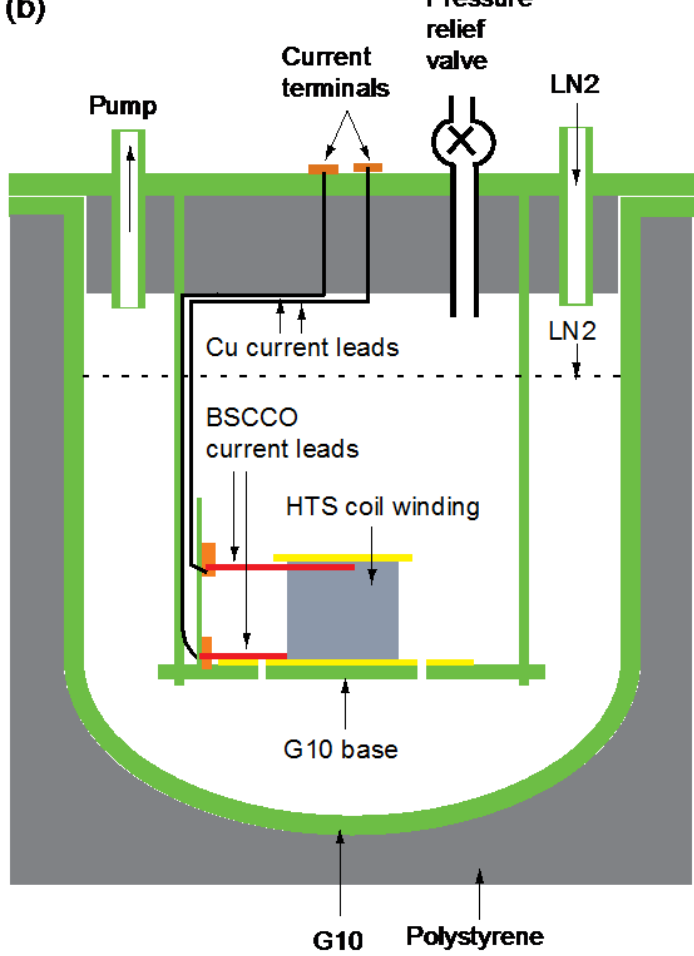

Figure 4. (a) Photo of the All-BSCCO assembly: the CW comprising four stacks and two EWs wound with BSCCO wire, (b) the schematic of measurement set-up. 


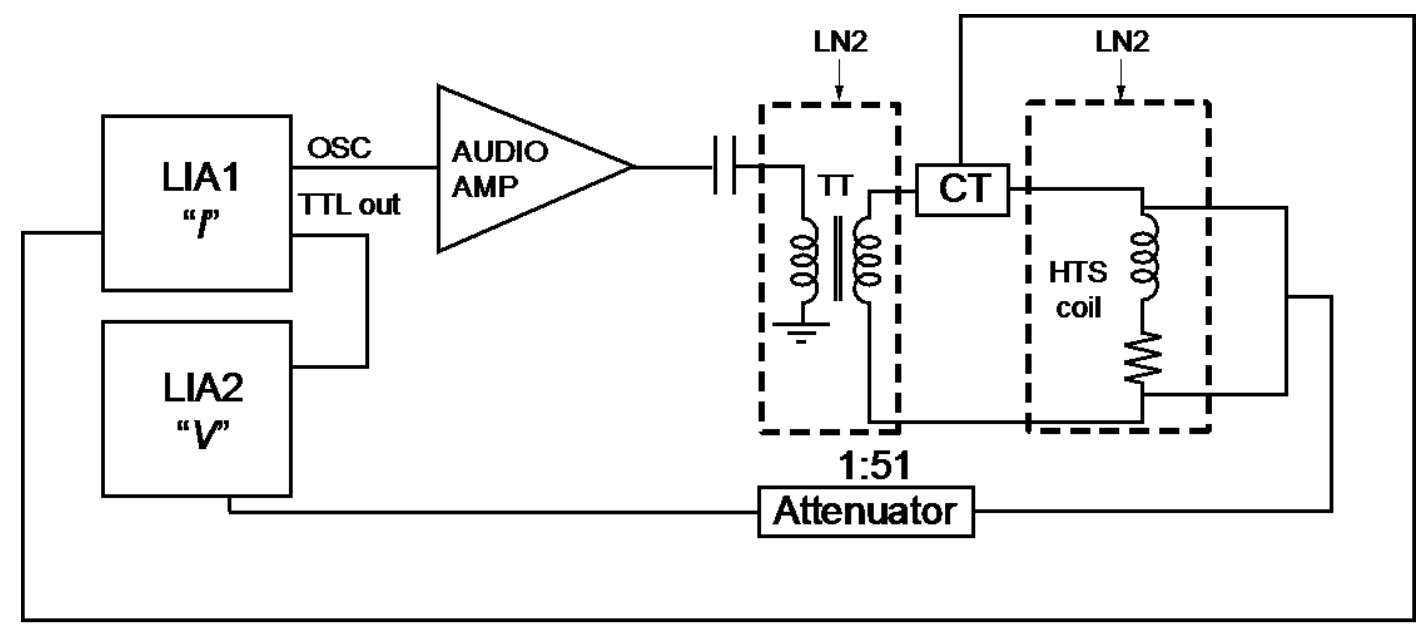

LIA: Lock-in-amplifier

TT: Toroidal transformer CT: Current tranformer LN2: Liquid nitrogen

Figure 5. Schematic of the AC loss measurement circuit 


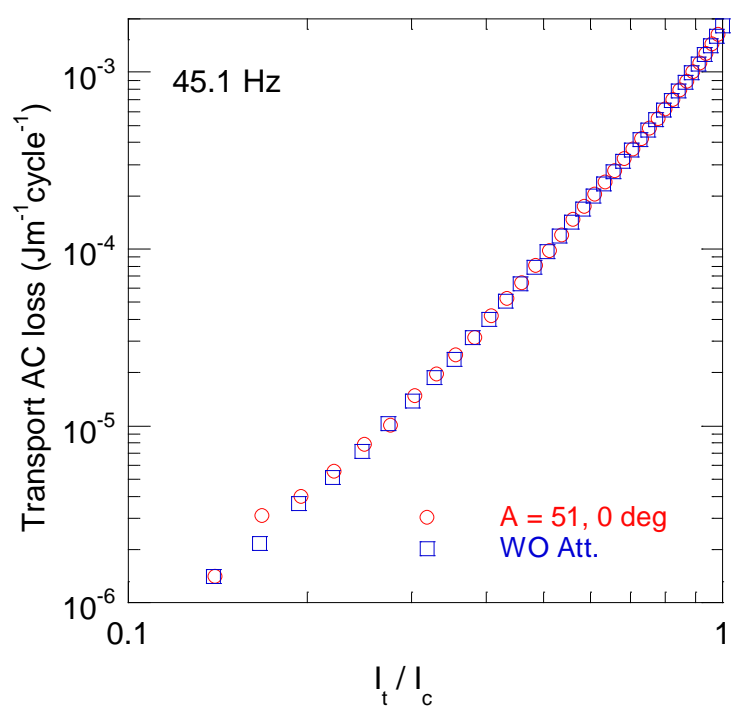

Figure 6. Comparison of transport AC loss results in an $82 \mathrm{~cm}$-long ReBCO wire with/without attenuator.

\section{Experimental results and discussion}

3.1 Coil $I_{\mathrm{c}}$ measurement

$E-I$ curves for the coil assemblies were measured at $77 \mathrm{~K}$ and $65 \mathrm{~K}$ and are shown in figure 7 . Table 2 shows

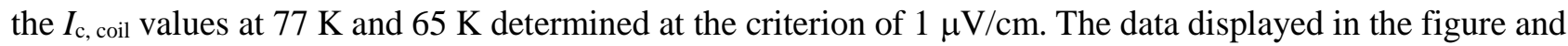
the table have the contact resistances arising from the soldering joints, listed in table 3 , between the DPCs subtracted. The $I_{\mathrm{c} \text {, coil }}$ value of the $\mathrm{BSCCO} / \mathrm{ReBCO}$ assembly is larger than the All-BSCCO assembly. This is because of the higher $I_{\mathrm{c}}$ of the wire used in YBCO EW compared to the wire employed in the BSCCO EW. The $I_{c}$, coil value of the All-ReBCO assembly is lower than the BSCCO/ReBCO assembly because the ReBCO $\mathrm{CW}$ employed a much smaller $I_{\mathrm{c}}$ wire than in the BSCCO CW. The lift factor of coil $I_{\mathrm{c}}$ at $65 \mathrm{~K}$ compared to 77

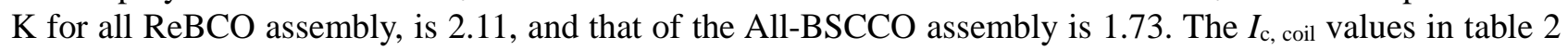
are used in Section 3.2 when comparing coil AC loss results with different $I_{\mathrm{c} \text {, coil }}$ values. 


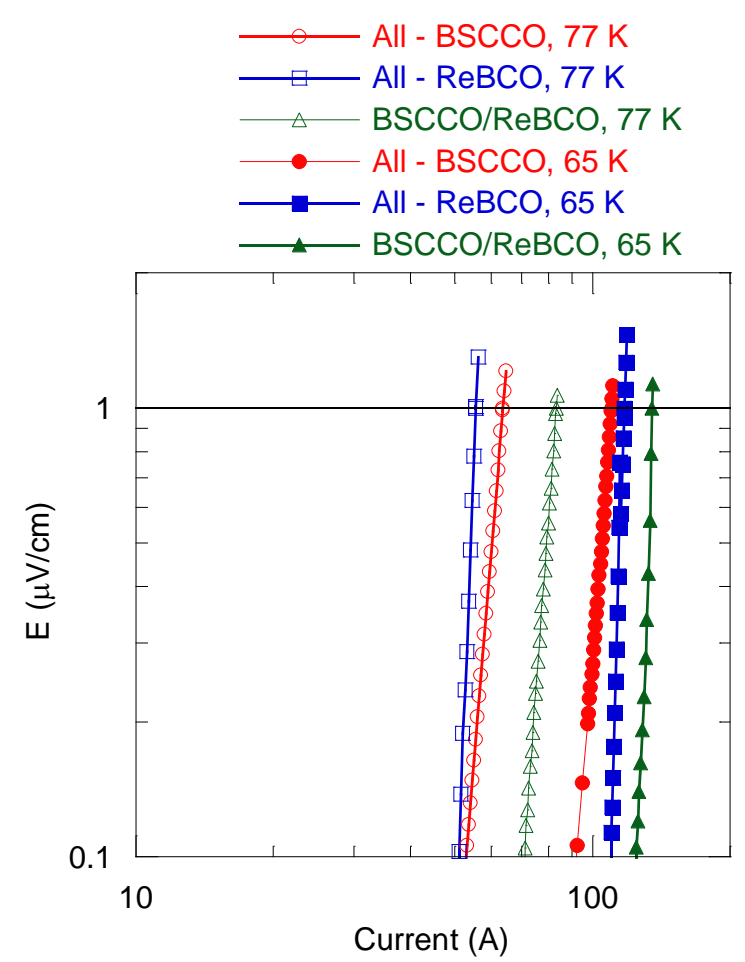

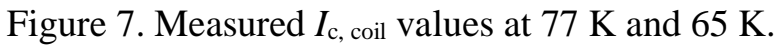

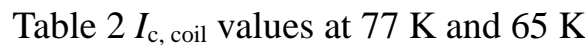

\begin{tabular}{cccc}
\hline Name of coil assembly & $I_{\mathrm{c}, \text { coil }}$ at $77 \mathrm{~K}(\mathrm{~A}) I_{\mathrm{c}, \text { coil }}$ at $65 \mathrm{~K}(\mathrm{~A})$ & $I_{\mathrm{c}, \text { coil } 65 \mathrm{~K} / I_{\mathrm{c}, \text { coil, } 77 \mathrm{~K}}}$ \\
All-BSCCO & 63.6 & 110.1 & 1.73 \\
All-ReBCO & 55.5 & 117.2 & 2.11 \\
BSCCO/ReBCO & 82.3 & 135.2 & 1.65 \\
\hline
\end{tabular}

Table 3 Contact resistance, $R_{\mathrm{c}}$ values at $77 \mathrm{~K}$ and $65 \mathrm{~K}$

Name of coil assembly $\quad R_{\mathrm{c}}$ at $77 \mathrm{~K}(\mu \Omega) \quad R_{\mathrm{c}}$ at $65 \mathrm{~K}(\mu \Omega)$

$\begin{array}{ccc}\text { All-BSCCO } & 1.21 & 1.0 \\ \text { All-ReBCO } & 4.96 & 4.56 \\ \text { BSCCO/ReBCO } & 3.92 & 3.6\end{array}$

3.2 AC loss measurement

3.2.1 AC loss in single wires

Figure 8(a) shows the measured transport AC loss plotted as a function of the applied current at various 
frequencies for a short length of AMSC BSCCO wire. The wire self-field $I_{\mathrm{c}}$ at $77 \mathrm{~K}$ is $156 \mathrm{~A}$, was cut from the same material used in the All-BSCCO coil assembly. In the figure, the loss calculated using the Norris ellipse model (N-e) and the strip model (N-s) are also plotted [25]. The measured AC loss per cycle is frequency independent, indicating the loss is hysteretic. The transport AC loss in the low current region deviates from the Norris ellipse calculation, which might be attributed to the spatial variation of local $J_{\mathrm{c}}$ [26]. The current dependence shown in figure 8(a) is consistent with a shell of higher $J_{\mathrm{c}}$ material and a core of lower $J_{\mathrm{c}}$ material within the elliptical envelope of coupled filaments typical of BSCCO powder-in-tube conductor. As the current amplitude approaches $I_{\mathrm{c}}$, the transport AC loss values tend to agree with the Norris ellipse model [27].

In figure 8(b), transport AC loss on a sample of SuNAM ReBCO wire at various frequencies is plotted as a function of the applied current. The wire is from the same material used for the EWs of the All-ReBCO coil assembly and the BSCCO/ReBCO assembly. The self-field $I_{\mathrm{c}}$ of the sample at $77 \mathrm{~K}$ was $210.5 \mathrm{~A}$. The Norris ellipse and strip model predictions are also shown. The lack of frequency dependency in the results suggests the $\mathrm{AC}$ loss is hysteretic. In the high current amplitude region, the measured transport AC loss agrees well with the Norris strip model. In the low current amplitude region, the measured AC loss lies between N-s and N-e, which is due to a lateral distribution of local $J_{\mathrm{c}}$ [28], with lower $J_{\mathrm{c}}$ material at the edges of the tape. Transport AC loss for a sample of the SuperPower wire (SCS 4050) used in the ReBCO CW was not measured. However, judging from measurements [14] done on a similar SuperPower wire, the AC loss characteristics are likely to be similar to those of the SuNAM ReBCO wire shown in figure 8(b).
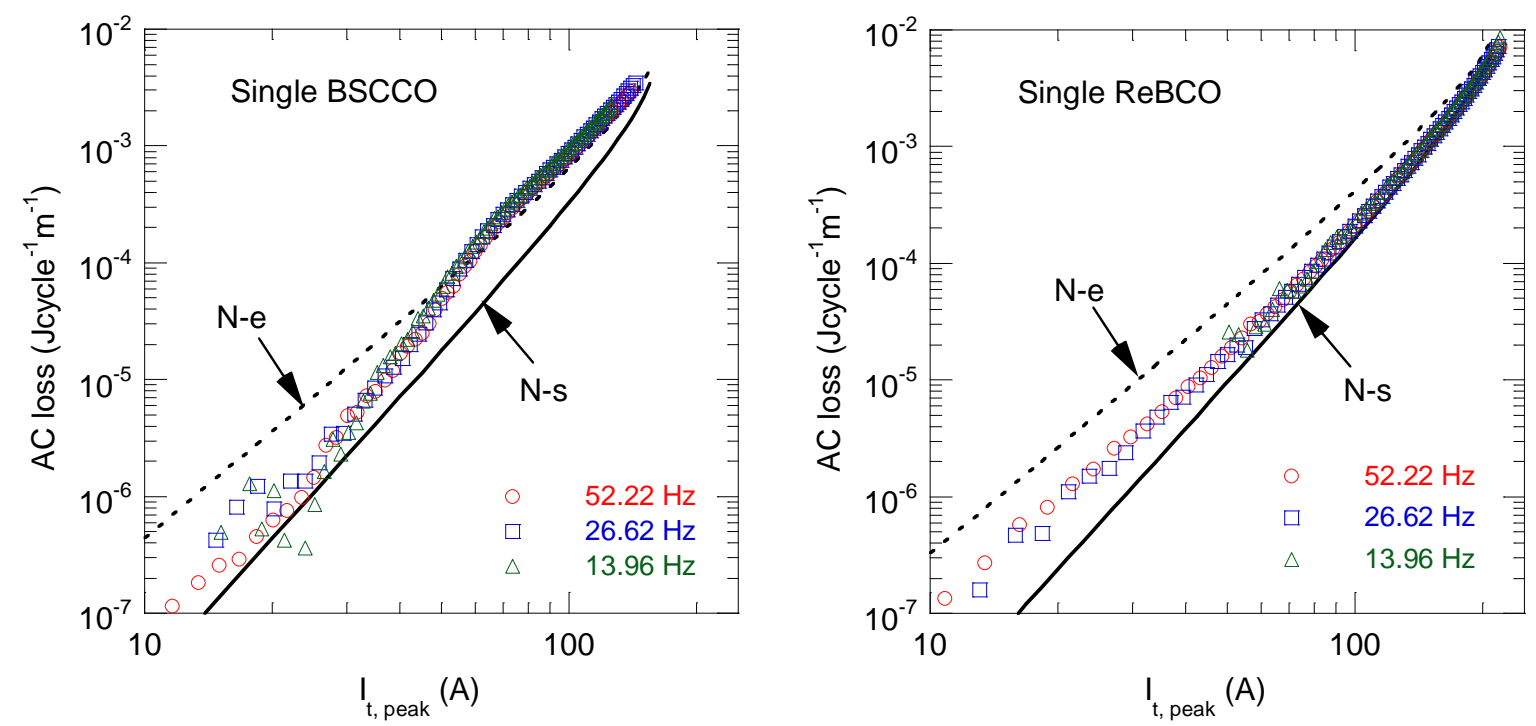

Figure 8. Measured transport AC loss in AMSC BSCCO and SuNAM ReBCO single wires, (a) BSCCO wire and (b) ReBCO wire for ReBCO EWs. In the figures, N-e and N-s denote Norris ellipse and strip models respectively.

\subsubsection{AC loss in the coil assemblies}

The loss contribution due to the contact resistance, $R_{\mathrm{c}}$, between the DPCs is negligible compared to the net AC loss in the superconductor assemblies. As an example figure 9 shows that the net loss in the All-ReBCO assembly at $77 \mathrm{~K}$ and $43.96 \mathrm{~Hz}$ is two orders of magnitude larger than the ohmic loss due to $R_{\mathrm{c}}$ shown in table 3. In the figures that follow, the AC loss in the coil assemblies is the net AC loss, having subtracted the ohmic loss component due to $R_{\text {c }}$.

Figures 10 and 11 show the measured AC loss in the coil assemblies at various frequencies, and at $77 \mathrm{~K}$ and $65 \mathrm{~K}$, plotted as a function of the current amplitude. In figures 10 (a) and (b), the measured AC loss for the All-BSCCO and All-ReBCO assemblies are compared with the results of single BSCCO and ReBCO wires (from figure 8) respectively. The measured loss per cycle for all coil assemblies display no evidence of frequency dependence which confirms the hysteretic nature of the loss. The AC loss curves for the different assemblies vary in slope. The power law dependency on applied current are approximately: All-BSCCO assembly, 2.3 to 2.05; All-ReBCO 2.7 to 3.0; BSCCO/ReBCO 2.9 to 2.4. The different power law dependence 
observed for BSCCO and ReBCO coils is in accord with previous reports [6, 10-15]. AC losses in the coil assemblies in units of $\mathrm{J} / \mathrm{m} /$ cycle are two to three orders of magnitude larger than that the loss in their respective single wires at $77 \mathrm{~K}$.

Figure 12 compares AC loss results in the coil assemblies measured at $77 \mathrm{~K}$ and $65 \mathrm{~K}$. The loss at $77 \mathrm{~K}$ is larger than at $65 \mathrm{~K}$. This can be explained by the change in $I_{\mathrm{c} \text {, coil }}$ values. Due to an increase in $I_{\mathrm{c} \text {, coil, }}$, the AC loss will be reduced in proportion to $I_{t} / I_{\mathrm{c}}$, coil, because the AC loss in the coil assemblies is in proportional to $\left(I_{t} / I_{c} \text {, coil }\right)^{2-4}$ which was also observed in this work. In the All-ReBCO assembly, the AC loss reduction due to lowering the temperature is the largest, as the ratio of $I_{\mathrm{c} \text {, coil }}$ at $65 \mathrm{~K}$ to that at $77 \mathrm{~K}$, the "lift factor", is highest as shown in table 2 .

Figure 13 compares the AC loss in the coil assemblies, at $77 \mathrm{~K}$ and $65 \mathrm{~K}$, normalised by $I_{\mathrm{c} \text {, coil }}$ squared as a function of coil current amplitude normalised by the $I_{\mathrm{c} \text {, coil. }}$ The $I_{\mathrm{c} \text {, coil }}$ values used are listed in table 3 . The scaled AC loss results at $77 \mathrm{~K}$ and $65 \mathrm{~K}$ then agree well with each other, the All-ReBCO and BSCCO/ReBCO assemblies within a few percent, and the All-BSCCO assembly to within 10-15\%. This implies AC loss in the assemblies can simply be scaled by the macroscopic coil $I_{\text {c }}$. Similarly, scaling AC loss by the overall wire/cable critical current has been reported in single wires and Roebel cables [24, 26, 29].

Theoretical justification for this scaling is provided by the expression [30], derived from Brandt and Indenbom [31] for total loss in a conductor, valid in the case of field-independent $J_{\mathrm{c}}$ :

$Q=k I_{\mathrm{c}}^{2} \Gamma(i, \beta)$ where $i=I / I_{\mathrm{c}}, \beta=H / H_{\mathrm{c}}, H_{\mathrm{c}}=J_{\mathrm{c}} t / \pi$, and $t$ is thickness of superconductor.

Since the local field in a coil is proportional to the coil current, the coil AC loss will be proportional to the product of $I_{\mathrm{c}}{ }^{2}$ and a function of $I / I_{\mathrm{c}}$ which will depend on the coil geometry. For field-independent $J_{\mathrm{c}}$ the coil critical current $I_{\mathrm{c} \text {, coil }}$ is the same as the conductor $I_{\mathrm{c}}$. This is plainly not the case for the coils here, as summarised in table 4.

Table $4 I_{\mathrm{c}, \text { coil }}$ values at $77 \mathrm{~K}$ compared to wire $I_{\mathrm{c}}$ and length weighted average wire $I_{\mathrm{c} \text {, ave }}$

\begin{tabular}{rccc}
\hline Coil assembly & $I_{\mathrm{c}, \text { coil }}(\mathrm{A})$ & Wire $I_{\mathrm{c}}$ a $(\mathrm{A})$ & $I_{\mathrm{c}, \text { coil }} / I_{\mathrm{c} \text {, ave }}$ \\
All-BSCCO & 63.6 & 156 & $41 \%$ \\
All-ReBCO & 55.5 & $86,223,132$ & $42 \%$ \\
BSCCO/ReBCO & 82.3 & $156,223,178$ & $46 \%$
\end{tabular}

\footnotetext{
${ }^{a}$ Comma-separated values are $I_{\mathrm{c}}$ values for the $\mathrm{CW}$ conductor, EW conductor, and the length-weighted average for the coil.
}

The All-BSCCO assembly retains only $41 \%$ of the $I_{\mathrm{c}}$ of the conductor, the other assemblies somewhat more, based on the average $I_{\mathrm{c}}$ of the conductor, so the reduction in $J_{\mathrm{c}}$ with local field, predominantly the radial field in the end turns of the assembly, is seen to have a major impact on $I_{c \text {, coil. }}$ E.g. the EWs in the All-BSCCO assembly are exposed to radial magnetic fields ranging from $50-125 \mathrm{mT}$, while the $\mathrm{CW}$ in the assembly is exposed to radial magnetic fields less than $50 \mathrm{mT}$ (see figure A in the Appendix). Given the strong influence of local field on the coil $I_{\mathrm{c}}$ it is perhaps surprising that the scaling of the $77 \mathrm{~K}$ and $65 \mathrm{~K}$ loss measurements with $I_{\mathrm{c} \text {, coil }}$ is so exact. 


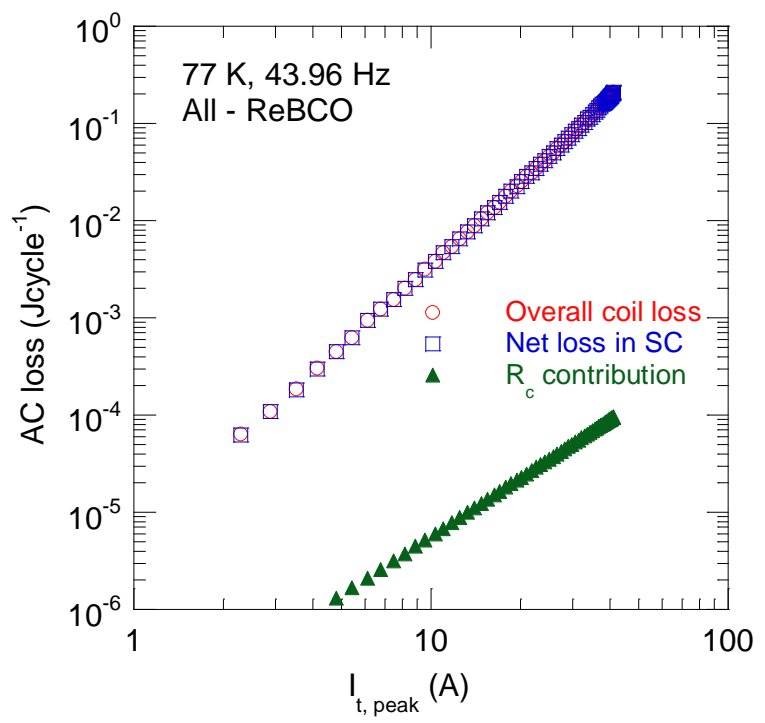

Figure 9. An example of loss contribution due to contact resistance, $R_{\mathrm{c}}$ in overall coil assembly AC loss.
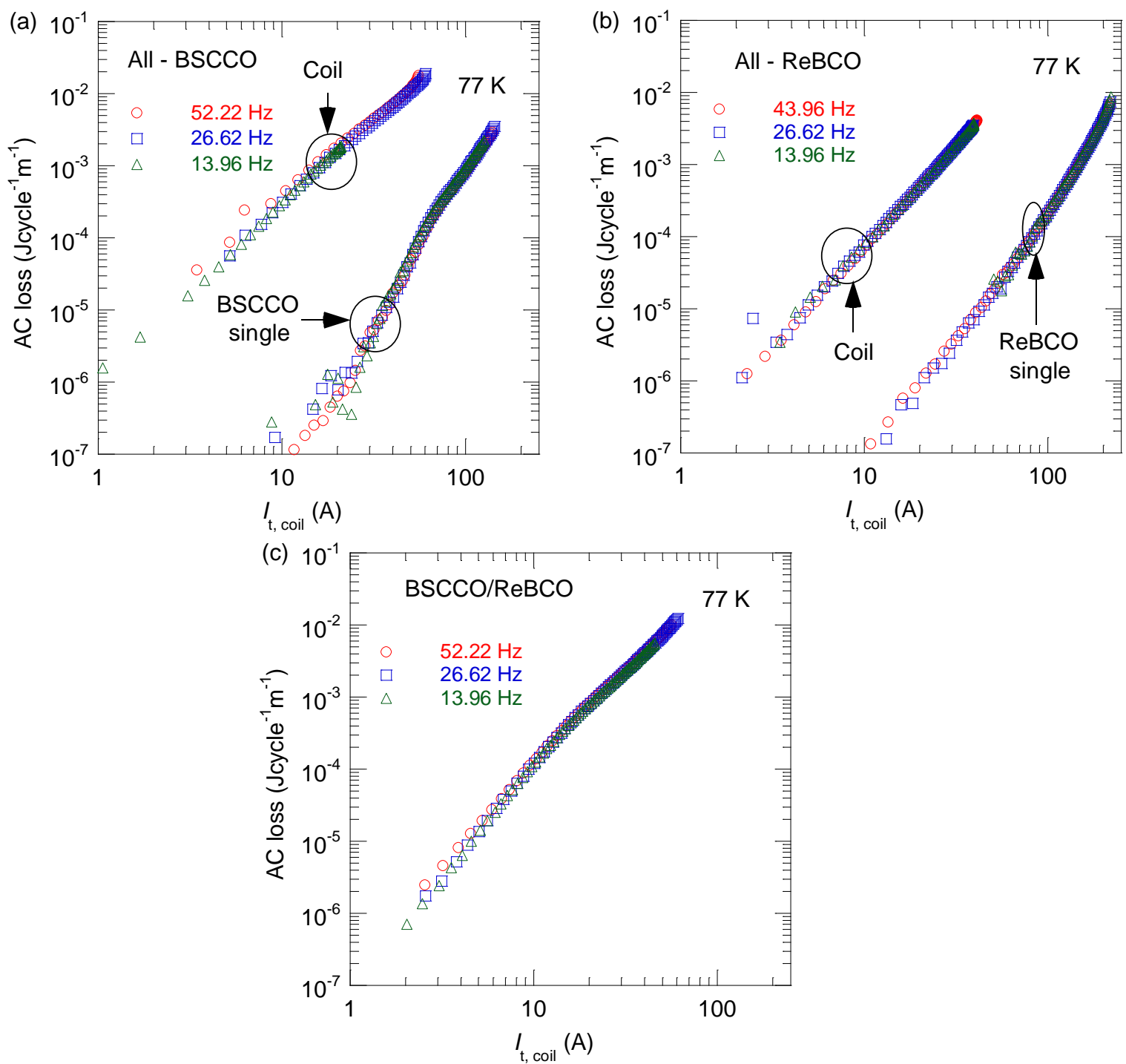

Figure 10. Measured coil AC loss results at $77 \mathrm{~K}$, (a) the All-BSCCO assembly, (b) the All-ReBCO assembly, and (c) the BSCCO/ReBCO assembly. 

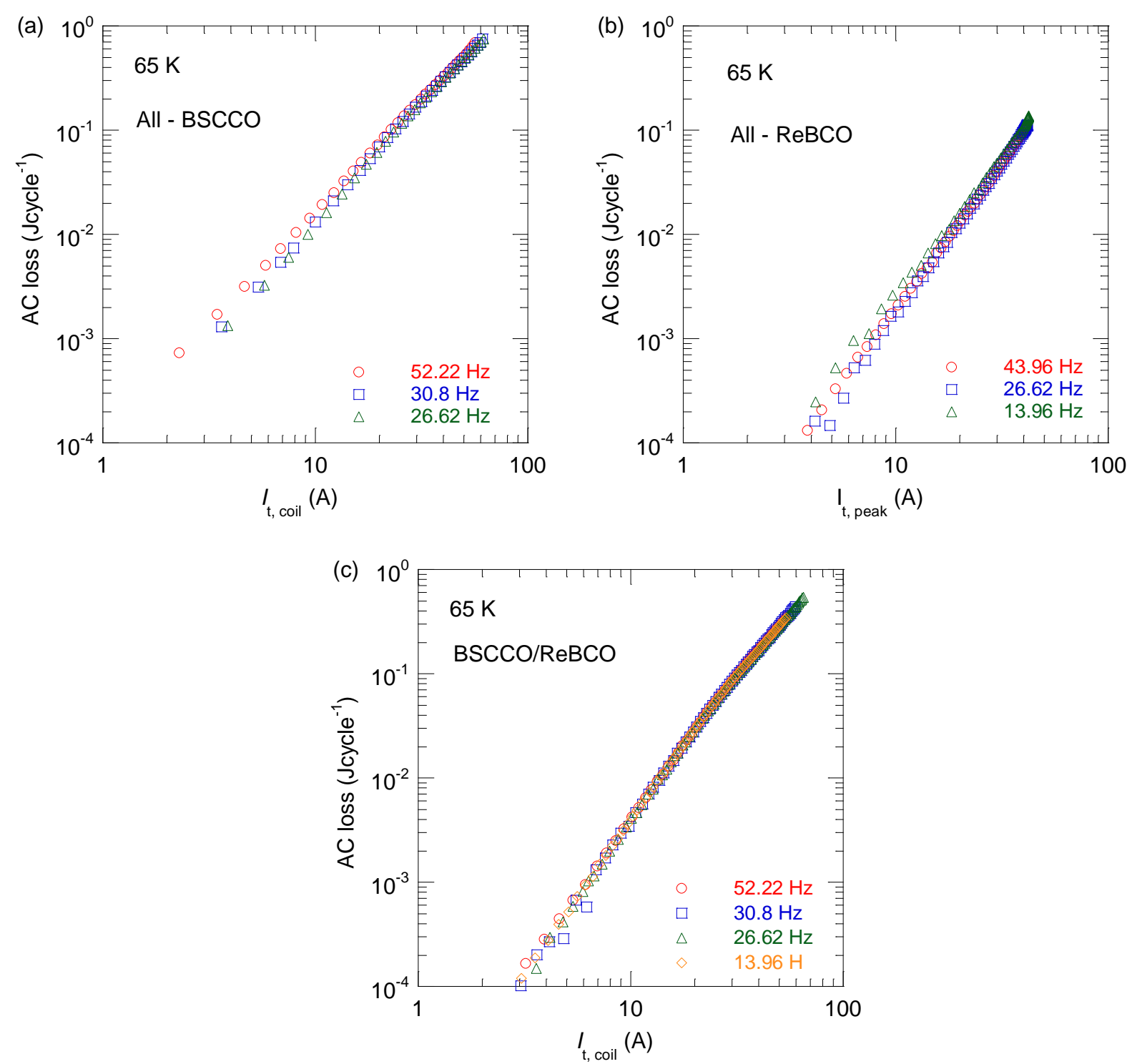

Figure 11. Measured coil AC loss results at $65 \mathrm{~K}$, (a) the All-BSCCO assembly, (b) the All-ReBCO assembly, and (c) the BSCCO/ReBCO assembly. 

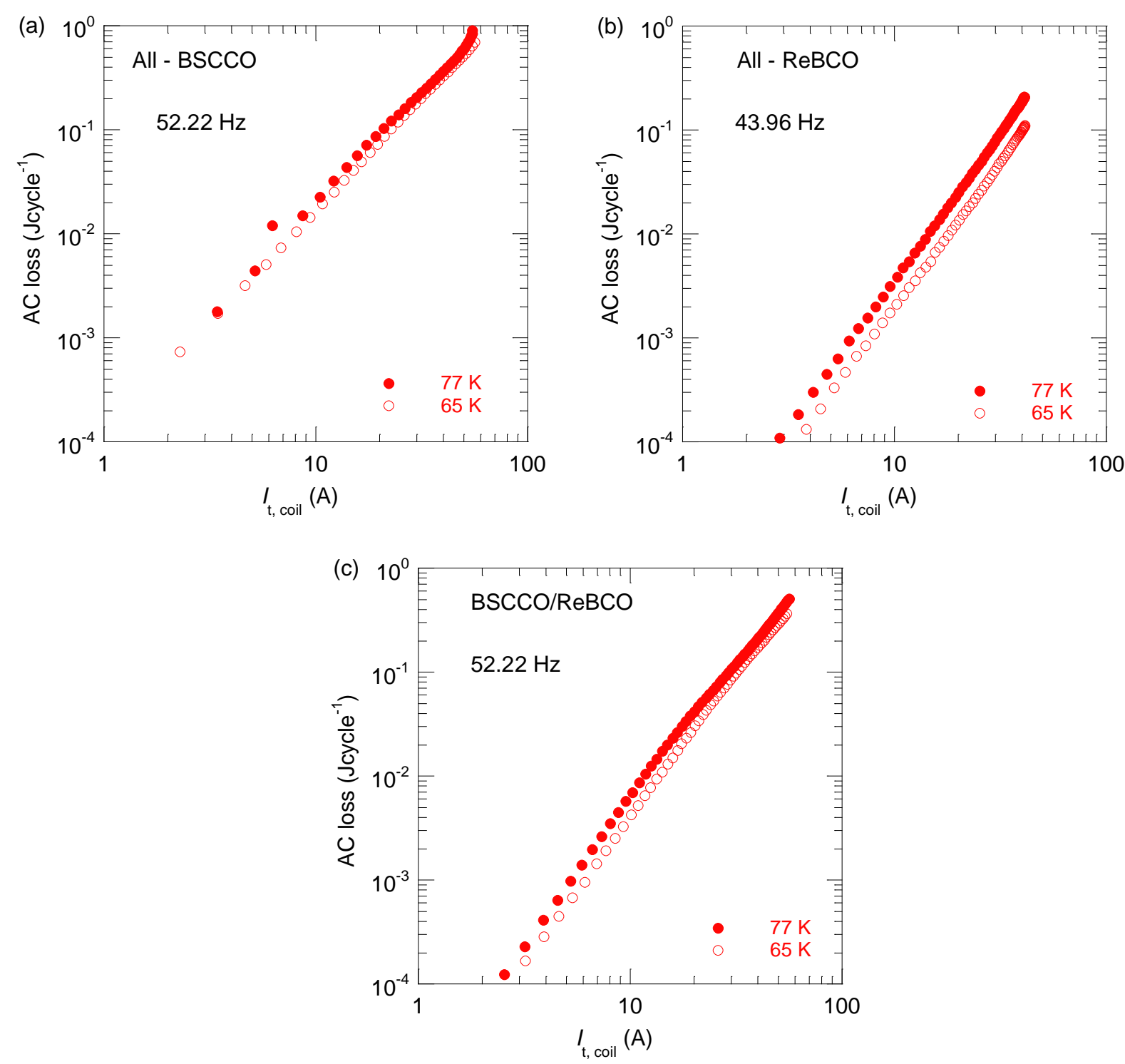

Figure 12. Comparison of measured coil AC loss at $77 \mathrm{~K}$ and $65 \mathrm{~K}$, (a) the All-BSCCO assembly, (b) the All$\mathrm{ReBCO}$ assembly, and (c) the BSCCO/ReBCO assembly. 

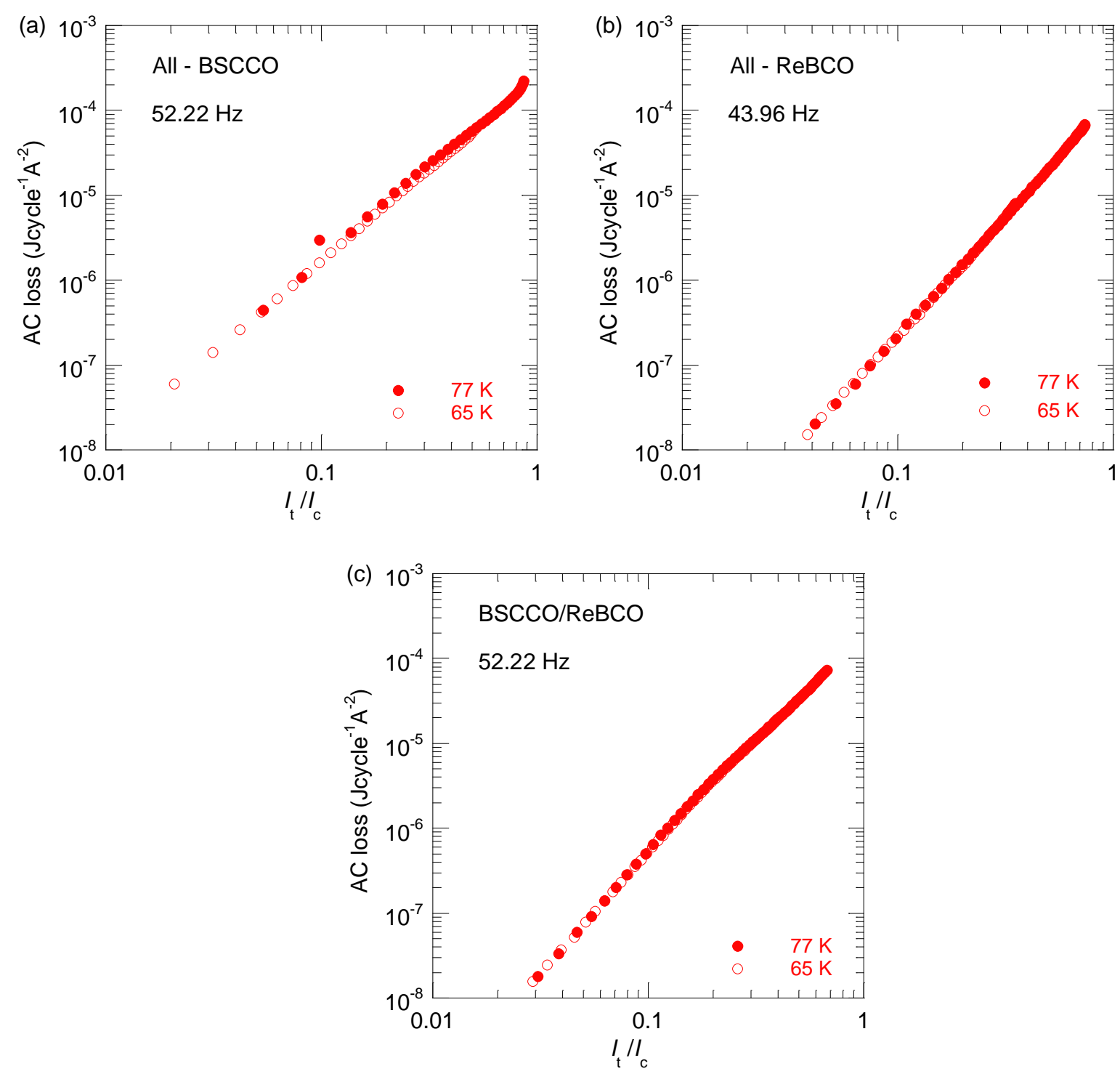

Figure 13. Comparison of $I_{\mathrm{c}}$ - normalised coil AC loss at $77 \mathrm{~K}$ and $65 \mathrm{~K}$, (a) the All-BSCCO assembly, (b) the All-ReBCO assembly, and (c) the BSCCO/ReBCO assembly.

\subsubsection{Comparison of AC loss in the coil assemblies}

Figure 14 compares the AC loss values for the assemblies at $77 \mathrm{~K}$. We compare first the All-BSCCO assembly and the BSCCO / ReBCO assembly, where these two assemblies share the same centre coil, BSCCO $\mathrm{CW}$. The AC loss for the BSCCO/ReBCO assembly is smaller by a factor of two over most of the current range compared to the All-BSCCO assembly. The only difference between these two assemblies is the wire used in the end windings. The YBCO EW has very similar geometry to BSCCO EW, implying that the difference is related to the different $I_{\mathrm{c}}$ values of the two conductors used in the EWs.

Next we compare AC loss values in the BSCCO/ReBCO assembly with those in the All-ReBCO assembly. The assemblies share the same end winding, ReBCO EW, but have different CWs: one uses BSCCO CW and the other uses ReBCO CW. The AC loss in the All-ReBCO assembly is smaller than the BSCCO/ReBCO assembly in the low coil current region, although the two curves tend to converge in the high current region. One reason why the AC loss values in the BSCCO/ReBCO assembly exceed than those in the All-ReBCO assembly could be the larger AC loss in BSCCO CW compared to the ReBCO CW due to the high axial field in the central section of the assembly. The greater thickness of the filamentary region of the BSCCO wire compared to the HTS film thickness of the ReBCO tape, around $155 \mu \mathrm{m}$ compared to $1 \mu \mathrm{m}$ for the coated 
conductor, means that the magnetic loss due to parallel (axial in the case of these windings) field can be larger for BSCCO.

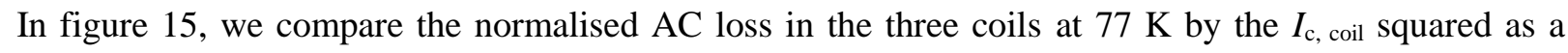
function of the applied coil current normalised by the coil $I_{\mathrm{c}}$. The AC loss in the BSCCO/ReBCO assembly is larger than the loss in the All-ReBCO assembly. Compared to figure 14, it cannot be said that the normalisation brings the three curves into close agreement. In fact, where the BSCCO and All-ReBCO curves previously appeared to converge, the scaling has increased the separation. A number of factors may have contributed to this. In coils where a single uniform conductor is used throughout, the AC loss in turns exposed to the strongest perpendicular/radial field is many times greater than the loss in turns near the centre of the assembly subjected to predominantly parallel/axial magnetic field. With the mixed windings used here, the loss in sections of low $I_{\mathrm{c}}$ wire away from the high radial fields near the ends of the assembly should contribute a larger fraction of the assembly loss. Other factors could be the larger parallel field AC loss of BSCCO wire compared to ReBCO wire, and the different characteristics of $J_{\mathrm{c}}(B)$ in the three wire types used in the assemblies.

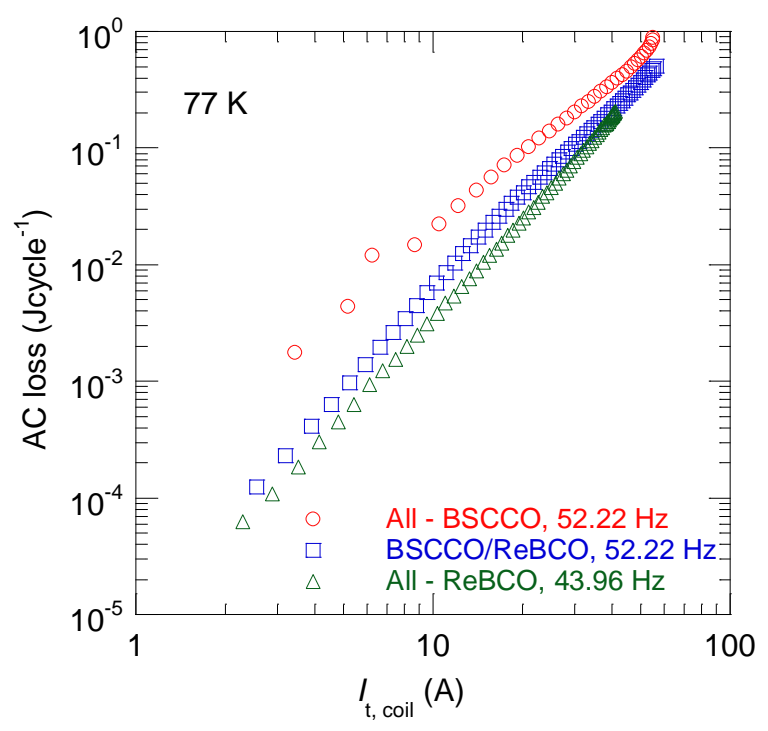

Figure 14. Comparison in AC loss values in the assemblies at $77 \mathrm{~K}$.

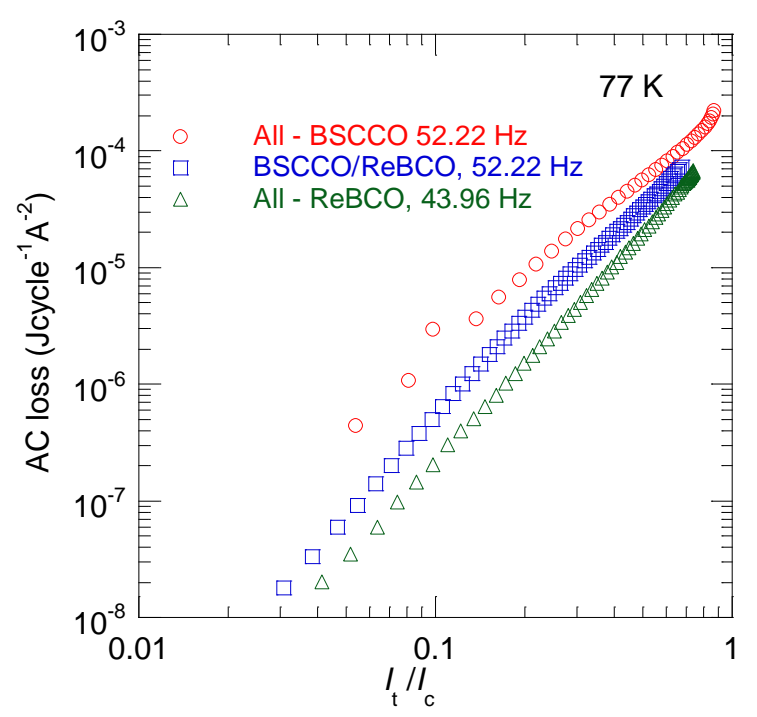

Figure 15. Comparison of the normalised AC loss results in the assemblies at $77 \mathrm{~K}$ - coil current normalised by $I_{\mathrm{c} \text {, coil }}$, AC loss normalised by $I_{\mathrm{c}}{ }^{2}$, coil. 


\subsection{Cost optimisation of HTS windings}

The cost components of an HTS AC power system most affected by the specified wire Ic are the wire cost, the cost of the cooling system, and the lifetime cost of losses taking account of cryocooler efficiency. In a modelling study of a 40 MVA HTS transformer [32] for example the lifetime cost of losses of was estimated to be around $35 \%$ of the capital cost of the transformer assuming a cost of losses typical of northern European markets (relatively high by world standards) and operation at rated power $100 \%$ of the time. In this case the cost of losses was roughly equal to the wire cost. In a more typical case: lifetime cost of losses 4 USD/W, load profile typical of a substation transformer, the cost of losses would be closer to 1/6th of the wire cost. In such a case, total cost is rather insensitive to AC loss, and one could with advantage use wire that was only $20 \%$ cheaper even if that meant doubling the AC loss. Direct cost of losses is however not the only determinant of the optimum wire $I_{\mathrm{c}}$. In the $40 \mathrm{MVA}$ transformer study, wire of a minimum $I_{\mathrm{c}}$ of $120 \mathrm{~A} / \mathrm{mm}$ at the operating temperature of $65 \mathrm{~K}$ was required to keep the total loss of the transformer within the $2.8 \mathrm{~kW}$ capacity of the chosen high efficiency cryocooler to avoid incurring the cost of a second cryocooler.

The modelling study did not however consider the possibility which is the subject of the current paper: that by using lower cost wire in parts of the windings where it can be used with relatively little impact on AC loss, the cost of wire for the device can be reduced. Comparing the AC loss of the All-BSCCO and $\mathrm{BSCCO} / \mathrm{ReBCO}$ windings, the $\mathrm{AC}$ loss of the All-BSCCO winding at $30 \mathrm{~A}$ is roughly halved by replacing $1 / 3$ of the wire by $\mathrm{ReBCO}$ wire with about $50 \%$ higher $I_{\mathrm{c}}$. In the present case substituting more expensive ReBCO wire for BSCCO would not be justified on a cost basis because the total loss of around $10 \mathrm{~W}$ contributes a negligible amount to lifetime operating costs. In the case of a larger device where wire cost and cost of losses are comparable it may be possible to substitute low $I_{\mathrm{c}}$ wire for part of the winding with little increase in AC loss and required cooling capacity. Depending on wire manufacturers' pricing, the resulting cost reduction could be significant.

Our results suggest however that BSCCO wire in the central sections of a winding, where it is exposed to large axial ac field, may contribute significantly to AC loss. Low cost/low $I_{\mathrm{c}} \mathrm{ReBCO}$ wire might be better used instead of BSCCO in the central sections of windings to lower total wire cost with relatively little effect on the cost of losses.

\section{Conclusion}

We have measured AC losses in three HTS coil assemblies at $77 \mathrm{~K}$ and $65 \mathrm{~K}$ which have hybrid coil structures consisting of one central winding (CW) and two end windings (EWs) wound with ReBCO and BSCCO wires with different self-field $I_{\mathrm{c}}$ values.

When the AC losses are normalised by the temperature dependent $I_{\mathrm{c} \text {, coil }}$ squared and plotted as a function of coil current normalised by the $I_{\mathrm{c} \text {, coil }}$ the measurements at $77 \mathrm{~K}$ and $65 \mathrm{~K}$ are scaled to a common curve. This implies the variation of $\mathrm{AC}$ loss with temperature in each of the coil assemblies scales with macroscopic coil critical current.

The HTS coil assembly made up of a BSCCO CW and ReBCO EWs with wire with high self-field $I_{\mathrm{c}}$ value has lower AC loss than the All-BSCCO assembly due to the increased $I_{\mathrm{c} \text {, coil. }}$ This means BSCCO wire can be used in the central section of an assembly to save wire cost whilst high $I_{\mathrm{c}} \mathrm{ReBCO}$ wires can be used in at the end-winding where the radial field is highest to reduce AC loss.

The All-ReBCO coil assembly has a lower AC loss compared to the BSCCO/ReBCO hybrid assembly which we attribute to a lowered $\mathrm{AC}$ loss in the $\mathrm{CW}$ where the parallel magnetic field component dominates. In an all ReBCO coil assembly wire with a lower self-field $I_{\mathrm{c}}$ can be used for the $\mathrm{CW}$ where magnetic field is dominated by parallel components, and hence wire cost can be reduced.

This work has practical implications for AC HTS coil design where both AC loss and cost are critical factors for applications.

\section{Acknowledgments}

The authors acknowledge Gennady Sidorov for development of specialist apparatus and Sarah Spencer for SEM images of AMSC BSCCO wire. 


\section{Appendix}

Figure A shows radial magnetic field components in the different part of the innermost and outermost layers of the All-BSCCO assembly along axial direction. The calculation was done using FEMM (finite element method magnetics) assuming each turn of the assembly carries a current of $55 \mathrm{~A}$.

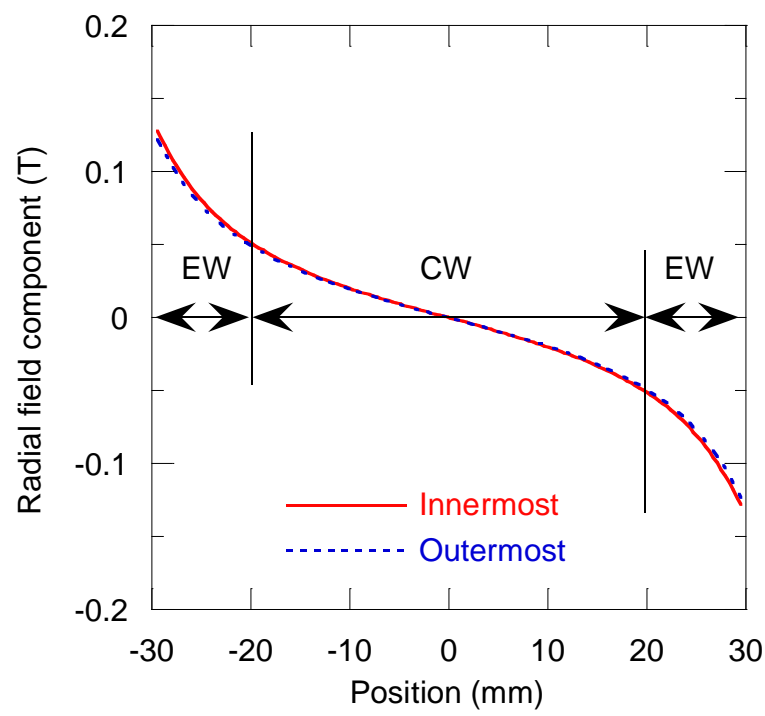

Figure A. A calculated variation of the radial field components of the innermost and outermost layers of the All-BSCCO assembly along the axial direction. 


\section{References}

[1] Kalsi S 2011 Application of high temperature superconductors to electric power equipment (IEEE Press) pp 102-106.

[2] Oomen M P, Nanke R and Leghissa M 2003 Modelling and measurement of ac loss in BSCCO/Agtape windings Supercond. Sci. Technol. $16339-54$.

[3] Pardo E, Staines M, Jiang Z and Glasson N 2015 AC loss modelling and measurement of superconducting transformers with coated-conductor Roebel-cable in low-voltage winding Supercond. Sci. Technol. 28114008.

[4] Zhang M, Yuan W, Kvitkovic J and Pamidi S 2015 Total AC loss study of 2G HTS coils for fully HTS machine applications Supercond. Sci. Technol. 28115011.

[5] Ainslie M D, Rodriguez-Zermeno V M, Hong Z, Yuan W, Flack T J and Coombs T A 2011 An improved FEM model for computing transport AC loss in coils made of RABiTS YBCO coated conductors for electric machines Supercond. Sci. Technol. 24045005.

[6] http://www.hts-110.com/

[7] Amemiya N, Enomoto N, Jiang Z, Kasai S, Saitoh T and Shiohara Y 2005 AC loss characteristics of coated conductor and perspective for its AC loss reduction Physica C 426-431 1267-75.

[8] Classssen J H 2006 An approximate method to estimate ac loss in tape-wound superconducting coils Appl. Phys. Lett. 88122512.

[9] Pardo E 2008 Modelling of coated conductor pancake coils with a large number of turns Supercond. Sci. Technol. 25065014.

[10] Yuan W, Ainslie M D, Xian W, Hong Z, Chen Y, Yan Y, Pei R and Coombs T 2011 Theoretical and experimental studies on $J_{\mathrm{c}}$ and AC losses of 2G HTS coils IEEE Trans. Appl. Supercond. 21 2441-4.

[11] Zhang M, Kvitkovic J, Kim J H, Kim C H, Pamidi S V and Coombs T A 2012 An Alternating current loss of second-generation high-temperature superconducting coils with magnetic and non-magnetic substrate Appl. Phys. Lett. 101102602.

[12] Jiang Z, Long N J, Badcock R A, Staines M, Slade R A, Caplin A D and Amemiya N 2012 AC loss measurements in pancake coils wound with $2 \mathrm{G}$ tapes and Roebel cable: dependence on spacing between turns/strands Supercond. Sci. Technol. 25035002.

[13] Pardo E, Šouc J and J Kováč 2012 AC loss in ReBCO pancake coils and stacks of them: modelling and measurement Supercond. Sci. Technol. 25035003.

[14] Jiang Z, Long N J, Staines M, Li Q, Slade R A, Amemiya N and Caplin A D 2012 Transport AC loss measurements in single-and two-layer parallel coated conductor arrays with low turn numbers IEEE Trans. Appl. Supercond. 228200306.

[15] Gömöry F, Šouc J, Pardo E, Seiler E, Soloviov M, Frolec L, Skarba M, Konopka P, Pekarčíková M and Janovec J 2012 IEEE Trans. Appl. Supercond. 228200306.

[16] Zermeno V M R and Grilli F 2014 3D modelling and simulation of 2G HTS stacks and coils Supercond. Sci. Technol. 27044025.

[17] Nyuyen D N, Kim C H, Kim J H, Pamidi S and Ashworth S P 2013 Electrical measurements of AC losses in high temperature superconducting coils at variable temperatures Supercond. Sci. Technol. 26 095001.

[18] Pardo E, Šouc J and Frolek 2015 Electromagnetic modelling of superconductors with a smooth current-voltage relation: variational principle and coils from a few turns to large magnets Supercond. Sci. Technol. 28044003.

[19] Queval L, Zermeno V M R and Grilli F 2016 Numerical models for ac loss calculation in large-scale applications of HTS coated conductors Supercond. Sci. Technol. 29024007.

[20] Kim J H, Kim C H, Iyyani G, Kvitkovic J and Pamidi S Transport AC loss measurements in superconducting coils 2011 IEEE Trans. Appl. Supercond. 21 3269-72.

[21] Yoon S and Kim J, Lee H, Han S and Moon S $201626 \mathrm{~T} 35 \mathrm{~mm}$ all- $\mathrm{GdBa}_{2} \mathrm{Cu}_{3} \mathrm{O}_{7-\mathrm{x}}$ multi-width noinsulation superconducting magnet Supercond. Sci. Technol. 29 04LT04.

[22] http://www.i-sunam.com/home/en_product, $1,3,1,1,1$

[23] http://www.superpower-inc.com/system/files/SP_2G+Wire+Spec+Sheet_2014_web_v1_0.pdf

[24] Jiang Z, Long N J, Staines M, Badcock R, Buckley R G, Talantsev E and Amemiya N 2013 The dependence of transport AC loss on temperature and DC parallel magnetic field in an eight-strand 
YBCO Roebel cable IEEE Trans. Appl. Supercond. 235402604.

[25] Norris W T 1970 Calculation of hysteresis losses in hard superconductors carrying ac: isolated conductors and edges of thin sheets J. Phys. D: Appl. Phys. 3 489-507.

[26] Yang Y, Hughes T and Beduz C 1997 Characteristics of the a.c. losses in Ag-sheathed PbBi2223 tapes Cryogenics 37 627-31.

[27] Ciszek M, Tsukamoto O and Amemiya N 1999 Angular dependence of AC transport losses in multifilamentary Bi-2223/Ag tape on external DC magnetic fields IEEE Trans. Appl. Supercond. $9817-$ 20.

[28] Amemiya N, Jiang Z, Li Z, Nakahata M, Kato T, Ueyama M, Kashima N, Nagaya S and Shiohara 2008 Transport losses in single and assembled coated conductors with textured-metal substrate with reduced magnetism Physica C 468 1718-22.

[29] Jiang Z, Staines M, Long N, Badcock R, Bumby C, Talantsev E, Hamilton K, Buckley R and Amemiya N 2014 The scaling of transport AC losses in Roebel cables with varying strand parameters Supercond. Sci. Technol. 27075007.

[30] Wolfbrandt A and Magnusson N 2002 AC losses in high-temperature superconducting tapes exposed to perpendicular magnetic fields combined with transport currents, Supercond. Sci. Technol. 15 (2002) 572-576.

[31] Brandt E H and Indenbom M 1993 Type-II-superconductor strip with current in a perpendicular magnetic field Phys. Rev. B 43 12893-12906.

[32] Staines M, Pardo E, Jolliffe L, Pannu M, Glasson N 2015 Prospects for HTS transformers in the grid: AC loss and economics, EUCAS 2015 poster 1A-LS-P-02.06, http://www.victoria.ac.nz/robinson/research/publications/Staines_EUCAS2015.pdf. 